\title{
ON PÓLYA FREQUENCY FUNCTIONS. III. THE POSITIVITY OF TRANSLATION DETERMINANTS WITH AN APPLICATION TO THE INTERPOLATION PROBLEM BY SPLINE CURVES( ${ }^{(1)}$
}

BY

\section{J. SCHOENBERG AND ANNE WHITNEY}

\section{INTRODUCTION}

1. A frequency function $\Lambda(x)$, i.e., a non-negative measurable function satisfying the inequalities

$$
0<\int_{-\infty}^{\infty} \Lambda(x) d x<\infty,
$$

is called a Pólya frequency function provided $\left({ }^{2}\right)$ it satisfies the following condition: For every two sets of increasing numbers

$$
x_{1}<x_{2}<\cdots<x_{n}, \quad y_{1}<y_{2}<\cdots<y_{n}, \quad n=1,2, \cdots,
$$

we have the inequality

$$
D \equiv \operatorname{det}\left\|\Lambda\left(x_{i}-y_{j}\right)\right\|_{1, n} \geqq 0 .
$$

For $n=1,(2)$ amounts to $\Lambda(x) \geqq 0$; for $n=2,(2)$ is equivalent to the convexity of $-\log \Lambda(x)$.

It was shown in [6] that the following two functions are Pólya frequency functions: 1. The normal frequency function whose Laplace transform is

$$
\frac{1}{2(\pi \gamma)^{1 / 2}} \int_{-\infty}^{\infty} e^{-8 x} e^{-(x-\delta)^{2} / 4 \gamma} d x=e^{\gamma s^{2}-\delta s}
$$

2. The "one-sided" exponential function

$$
\lambda(x)=\left\{\begin{array}{lll}
e^{-x} & \text { if } & x \geqq 0 \\
0 & \text { if } \quad x<0
\end{array}\right.
$$

of transform

Presented to the Society, April 30, 1949 under the title On the positivity of translation determinants for Pólya frequency functions; received by the editors April 4, 1952.

(1) This work was performed on a National Bureau of Standards contract with the University of California at Los Angeles and was sponsored in part by the Office of Scientific Research, USAF. Miss Whitney's contribution to this paper was accepted by the Graduate School of the University of Pennsylvania in partial fulfilment of the requirement for the Ph.D. degree. For a brief summary see [7] in the list of references at the end of this paper.

(2) In this paper we use the term "provided" in the sense of "if and only if." 


$$
\int_{-\infty}^{\infty} e^{-s x} \lambda(x) d x=\frac{1}{1+s}, \quad(\mathrm{R} s>-1) .
$$

If $\delta \gtrless 0$, we obtain the formula

$$
\frac{1}{|\delta|} \int_{-\infty}^{\infty} e^{-s x} \lambda\left(\frac{x}{\delta}+1\right) d x=\frac{e^{\delta s}}{1+\delta s}, \quad\left(-|\delta|^{-1}<\mathrm{R} s<|\delta|^{-1}\right),
$$

giving the transform of the one-sided exponential, of mean zero and variance $\delta^{2}$, which is descending if $\delta>0$ and ascending if $\delta<0$.

It was also shown in [6] that the convolution of Pólya frequency functions again leads to such functions. Moreover, that the most general Pólya frequency function $\Lambda(x)$ is obtained, up to a multiplicative positive constant, by convoluting the normal function (3) with a finite or infinite sequence of one-sided exponentials (5) which are such that the sum $\sum \delta_{\nu}^{2}$ of their variances converges. In other terms: the Laplace transform of a Pólya frequency function $\Lambda(x)$ converges in a vertical strip containing the origin and has there the form

$$
\int_{-\infty}^{\infty} e^{-x s} \Lambda(x) d x=\frac{1}{\Psi(s)}
$$

where $\Psi(s)$ is an entire function of the form

$$
\begin{aligned}
\Psi(s)= & C e^{-\gamma s^{2}+\delta s} \prod_{\nu=1}^{\infty}\left(1+\delta_{\nu} s\right) e^{-\delta_{\nu s}} \\
& \left(C>0, \gamma \geqq 0, \delta, \delta_{\nu} \text { real, } 0<\gamma+\sum \delta_{\nu}^{2}<\infty\right) .
\end{aligned}
$$

We single out the special case when $\gamma=0$ and $\sum\left|\delta_{\nu}\right|<\infty$. Except for a trivial exponential factor which can be absorbed into the integral by means of a shift in the variable $x$, our function $\Psi$ is of genus zero. We may therefore assume $\Lambda(x)$ to have the transform

$$
\int_{-\infty}^{\infty} e^{-x s} \Lambda(x) d x=\prod_{\nu=1}^{\infty} \frac{1}{1+\delta_{\nu} s}, \quad\left(\delta_{\nu} \text { real, } \sum\left|\delta_{\nu}\right|<\infty\right) .
$$

These two types of transforms, (8) and (6), (7), will be referred to below as Case 1 and Case 2, respectively.

The present paper is divided into two sections. In $\S 1$ we answer (Theorem 1 below) the following question: Given a Polya frequency function $\Lambda(x)$ and a set of $2 n$ numbers (1), how can we decide when the determinant $D$, defined by (2), is actually positive? As an application of our answer to this question we solve in $\$ 2$ the general problem of interpolation by so-called spline curves which were introduced in 1946 by one of us [5] for the purpose of approximation of infinitely many equidistant data. 


\section{The POSITIVITY OF TRANSLATION DETERMINANTS}

2. The following theorem answers our question.

Theorem 1. Let $\Lambda(x)$ be a Poblya frequency function of transform (8) (Case 1), or (6), (7) (where $\gamma>0$, or $\gamma=0$ and $\sum\left|\delta_{\nu}\right|=\infty$ ) (Case 2). Let the numbers (1) be given and let

$$
D=\operatorname{det}\left\|\Lambda\left(x_{i}-y_{j}\right)\right\|_{1, n} .
$$

Case $1\left(^{3}\right)$. Let $k$ be the number of positive $\delta_{y}$ and $h$ the number of negative $\delta_{\nu}(0 \leqq k \leqq \infty, 0 \leqq h \leqq \infty, k+h>1)$. Then $D>0$ if and only if we have the inequalities

$$
x_{v-k}<y_{v}<x_{v+h}
$$$$
\text { for } \nu=1, \cdots, n \text {, }
$$

with the convention that

$$
x_{r}=\left\{\begin{array}{llr}
-\infty & \text { if } & -\infty \leqq r<1, \\
+\infty & \text { if } & n<r \leqq+\infty .
\end{array}\right.
$$

Case 2. We always have $D>0$.

ExAmples. 1. It is clear from (1.3) that some of the inequalities (1.2) are automatically satisfied. Thus, if $k \geqq n$ and $h \geqq n$, then again $D>0$, no matter what the numbers (1) are. This is true for all $n$ if $k=h=0$. If $k>0$ and $h>0$ we obtain, for $n=1$, the fact that $\Lambda(x)>0$, for all $x$.

2. The function $\Lambda(x)=\exp (-|x|)$ of transform

$$
\int_{-\infty}^{\infty} e^{-x s} e^{-|x|} d x=\frac{2}{1-s^{2}} \quad(-1<\mathrm{R} s<1)
$$

illustrates Case 1 with $k=h=1$. From (1.2) we learn that $D>0$, provided $x_{\nu-1}<y_{\nu}<x_{\nu+1}(\nu=1, \cdots, n)$, or

$$
y_{1}<x_{2}, x_{1}<y_{2}<x_{3}, x_{2}<y_{3}<x_{4}, \cdots, x_{n-2}<y_{n-1}<x_{n}, x_{n-1}<y_{n} .
$$

In this particular case we mention as a curiosity that $D$ may be evaluated explicitly by the following formula, valid for any set (1),

$$
\operatorname{det}\left\|e^{-\left|x_{i}-y_{j}\right|}\right\|_{1, n}=e^{-\Sigma x_{i}-\Sigma y_{j}} \prod_{i=1}^{n}\left\{e^{2 \min \left(x_{i}, y_{i}\right)}-e^{2 \max \left(x_{i-1}, y_{i-1}\right)}\right\}_{+},
$$

where the subscript " + " indicates the truncated function

$$
x_{+}=\left\{\begin{array}{lll}
x & \text { if } & x \geqq 0 \\
0 & \text { if } & x<0 .
\end{array}\right.
$$

(3) The Case 1 of Theorem 1 is related, without inclusion either way, to a theorem of $M$. Krein and G. Finkelstein [4], concerning the Green's function of linear differential operators. Concerning this subject see the comprehensive treatise [1] by Gantmakher and Krein. 
3. The function

$$
\Lambda(x)=e^{-x} x_{+}^{k}
$$

of transform

$$
\int_{0}^{\infty} e^{-x s} e^{-x} x^{k} d x=\frac{k !}{(1+s)^{k+1}}
$$

corresponds to Case 1 with $h=0$ and $k+1$ positive $\delta_{\nu}$, all unity. Cancellation of (positive) exponential factors from the rows and columns of $D$ leads to the following:

CoRollary 1. Let $k$ be a natural integer. If $x_{1}<x_{2}<\cdots<x_{n}, y_{1}<y_{2}$ $<\cdots<y_{n}$, then

$$
D=\operatorname{det}\left\|\left(x_{i}-y_{j}\right)_{+}^{k}\right\|>0
$$

if and only if the inequalities

$$
x_{\nu-k-1}<y_{\nu}<x_{\nu}
$$$$
\nu=1, \cdots, n,
$$

$\operatorname{hold}\left({ }^{4}\right)$.

This is our special result to be applied in $\$ 2$.

\section{A proof of Theorem 1, Case 1}

3. We begin by noticing that the simplest of all cases, namely $k+h=1$, is not covered by Theorem 1 . Although almost trivial and unimportant by itself, it happens to be fundamental for what follows. So let us assume that $k=1, h=0$, in (8), hence $\Lambda(x)$ to be the one-sided exponential

$$
\Lambda(x)=\frac{1}{\delta} \lambda\left(\frac{x}{\delta}\right),
$$

Now $\lambda(x)=e^{-x} \cdot x_{+}^{0}$, where $x_{+}^{0}=1$ if $x \geqq 0,=0$ if $x<0$. Thus $D$ $=\operatorname{det}\left\|\Lambda\left(x_{i}-y_{j}\right)\right\|>0$ provided $D^{\prime}=\operatorname{det}\left\|\left(x_{i}-y\right)_{+}^{0}\right\|>0$. A moment's reflection will show that $D^{\prime}>0$, provided all elements of $D^{\prime}$ are $=1$, except those above its main diagonal which are $=0$. By the definition of $x_{+}^{0}$ these conditions amount to the inequalities

$$
y_{1} \leqq x_{1}<y_{2} \leqq x_{2}<\cdots<y_{n-1} \leqq x_{n-1}<y_{n} \leqq x_{n},
$$

or

$$
x_{\nu-1}<y_{\nu} \leqq x_{\nu} \quad \text { for } \nu=1, \cdots, n \text {. }
$$

(4) The nonvanishing of determinants of the form (1.7) appears explicitly as an assumption in some of the theorems of Chapter 9 in S. Mandelbrojt's Dirichlet series, Rice Institute Pamphlet, vol. 31, No. 4, October, 1944. 
We prove next a special case of Case $1(h=0,1<k<\infty)$ which, for convenience, we state as:

Lemma 1. If $\Lambda(x)$ has the transform

$$
\int_{-\infty}^{\infty} e^{-x s} \Lambda(x) d x=\prod_{\nu=1}^{k} \frac{1}{1+\delta_{\nu} s}, \quad \delta_{\nu}>0 \text { for all } \nu ; k>1,
$$

then

$$
D=\operatorname{det}\left\|\Lambda\left(x_{i}-y_{j}\right)\right\|>0
$$

if and only if

$$
x_{v-k}<y_{v}<x_{\nu},
$$$$
\nu=1, \cdots, n \text {. }
$$

Proof. Let $\Lambda_{1}(x)$ and $\Lambda_{2}(x)$ have the transforms

$$
\prod_{\nu=1}^{k-1} \frac{1}{1+\delta_{\nu} s} \text { and } \frac{1}{1+\delta_{k} s}
$$

respectively. Thus $\Lambda(x)$ is the convolution of $\Lambda_{1}(x)$ with $\Lambda_{2}(x)$. From [6, Lemma 5], we recall the identity

$$
\begin{aligned}
\operatorname{det}\left\|\Lambda\left(x_{i}-y_{j}\right)\right\|= & \frac{1}{n !} \int_{-\infty}^{\infty} \cdots \int \operatorname{det}\left\|\Lambda_{1}\left(x_{i}-t_{j}\right)\right\| \\
& \cdot \operatorname{det}\left\|\Lambda_{2}\left(t_{i}-y_{j}\right)\right\| d t_{1} \cdots d t_{n},
\end{aligned}
$$

which, in view of the symmetry of the integrand in the variables $t_{1}, \cdots, t_{n}$, we may write as

$$
\begin{aligned}
\operatorname{det}\left\|\Lambda\left(x_{i}-y_{j}\right)\right\| & =\int_{t_{1}<t_{2}<\cdots<t_{n}} \cdots \int \operatorname{det}\left\|\Lambda_{1}\left(x_{i}-t_{j}\right)\right\| \\
& \cdot \operatorname{det}\left\|\Lambda_{2}\left(t_{i}-y_{j}\right)\right\| d t_{1} \cdots d t_{n} \\
& =\int \cdots \int D_{1} D_{2} d t_{1} \cdots d t_{n} .
\end{aligned}
$$

Notice that $D_{1} \geqq 0$ and $D_{2} \geqq 0$, for all $(t), \Lambda_{1}$ and $\Lambda_{2}$ being Pólya frequency functions. $D_{2}$ is continuous in $(t)$ except possibly on the hyperplanes $t_{i}=y_{j}$. $D_{1}$ is continuous in $(t)$ if $k>2$ and possibly discontinuous on $t_{j}=x_{i}$ if $k=1$. We conclude that the integral (1.14) is positive if and only if there exists a point $(t)$, with $t_{1}<\cdots<t_{n}$, in a neighborhood of which both factors $D_{1}, D_{2}$ are positive. By (1.10), this is the case for $D_{2}$ provided

$$
t_{\nu-1}<y_{\nu}<t_{\nu}, \quad \nu=1, \cdots, n .
$$

Using induction by $k$ and assuming Lemma 1 to hold for $k-1$, rather than $k$, we see that $D_{1}>0$, at $(t)$ and near it, provided 


$$
x_{\nu-k+1}<t_{\nu}<x_{\nu}
$$$$
\nu=1, \cdots, n .
$$

Thus (1.14) is positive provided (1.15) and (1.16) have a common solution of increasing $t$ 's. Inspecting the bounds imposed on $t_{\nu}$ by (1.15) and (1.16), we find the necessary inequalities

$$
\max \left(x_{\nu-k+1}, y_{v}\right)<\min \left(x_{v}, y_{v+1}\right),
$$$$
\nu=1, \cdots, n .
$$

These, however, are also sufficient, for then the open intervals of the $t$-axis

$$
I_{\nu}: \max \left(x_{\nu-k+1}, y_{\nu}\right)<t<\min \left(x_{\nu}, y_{\nu+1}\right), \quad \nu=1, \cdots, n,
$$

form a sequence of nonoverlapping and advancing intervals, for increasing $\nu$, in view of the obvious inequalities $\min \left(x_{v}, y_{v+1}\right) \leqq \max \left(x_{v-k+2}, y_{v+1}\right)$. Choose $t_{\nu} \in I_{\nu}$ and both (1.15), (1.16) will be satisfied. By (1), the inequalities (1.17) are equivalent to

$$
x_{\nu-k+1}<y_{\nu+1}, \quad y_{\nu}<x_{\nu}, \quad \text { or } \quad x_{\nu-k}<y_{\nu}, \quad y_{\nu}<x_{\nu},
$$

which are identical with (1.13). As the same argument applies if $k=2$, the proof by induction is complete.

REMARK. If we replace in Lemma 1 the assumption $\delta_{\nu}>0$, for all $\nu$, by

$$
\delta_{\nu}<0, \quad \nu=1, \cdots, n,
$$

we have a problem which reduces to the previous case as follows: Replacing $s$ by $-s$ in (1.11) we obtain

$$
\mathrm{II} \frac{1}{1+\left|\delta_{\nu}\right| s}=\int_{-\infty}^{\infty} e^{s x} \Lambda(x) d x=\int_{-\infty}^{\infty} e^{-s x} \Lambda(-x) d x .
$$

This interchanges the roles of $x_{i}$ and $y_{j}$, and (1.12) holds now provided

$$
y_{\nu-k}<x_{\nu}<y_{\nu}, \quad \nu=1, \cdots, n .
$$

4. We may now complete a proof of Case 1 by a brief discussion of a few subcases:

Proof of Case 1 for finite positive $k$ and $h$. Let the transform (8) be rational, there being $k$ positive and $h$ negative $\delta_{\nu}$. Let $\Lambda_{1}$ and $\Lambda_{2}$ have the transforms

$$
\prod_{\nu=1}^{k} \frac{1}{1+\delta_{\nu} s}, \quad \delta_{\nu}>0, \quad \prod_{\nu=1}^{h} \frac{1}{1+\delta_{k+\nu} s}, \quad \delta_{k+\nu}<0,
$$

respectively. Then $\Lambda=\Lambda_{1} * \Lambda_{2}$ and (1.14) holds. By Lemma 1 and its counterpart (1.19), (1.20), a point $(t)$ and its neighborhood will produce a positive integrand in (1.14) provided we have

$$
x_{v-k}<t_{v}<x_{v}, \quad y_{p-k}<t_{v}<y_{p},
$$$$
\nu=1, \cdots, n \text {. }
$$

These require that 


$$
\max \left(x_{v-k}, y_{v-h}\right)<\min \left(x_{v}, y_{v}\right),
$$$$
\nu=1, \cdots, n,
$$

which again are also sufficient. Indeed, if (1.22) hold, the intervals

$$
I_{v}^{\prime}: \max \left(x_{v-k}, y_{v-h}\right)<t<\min \left(x_{v}, y_{v}\right) \quad \nu=1, \cdots, n,
$$

which may well overlap, have advancing right-hand end points (by (1)), hence an increasing sequence $\left\{t_{\nu}\right\}$ with $t_{\nu} \in I_{\nu}^{\prime}$ is assured. Finally (1.22) amount to $x_{\nu-k}<y_{\nu}, y_{\nu-h}<x_{\nu}$, which in turn are equivalent to (1.2).

Proof of Case 1 for $k=\infty, h=0$. Let the transform of $\Lambda(x)$ be (8) with all $\delta_{v}>0$. We are to show that $D=\operatorname{det}\left\|\Lambda\left(x_{i}-y_{j}\right)\right\|>0$ if and only if

$$
y_{\nu}<x_{\nu}, \quad \nu=1, \cdots, n .
$$

The necessity of these inequalities is seen directly as follows: In the present case of positive $\delta_{v}$ 's, (8) is an ordinary Laplace integral (see [6, Art. 11]), i.e., $\Lambda(x)=0$ if $x \leqq 0$. But then $x_{\mu} \leqq y_{\mu}$, for some $\mu$, implies $x_{i} \leqq y_{j}$ for all $(i, j)$ such that $i \leqq \mu, j \geqq \mu$. Hence $\Lambda\left(x_{i}-y_{j}\right)=0$, for such $(i, j)$, and $D$ vanishes, for instance by Laplace's expansion theorem.

In order to prove the sufficiency of (1.23), we choose a number $k^{\prime}>n$ and write $\Lambda=\Lambda_{1} * \Lambda_{2}$, where $\Lambda_{1}$ corresponds to the first $k^{\prime}$ factors of the product (8) and $\Lambda_{2}$ to all remaining ones. In (1.14), $D_{1}>0$ at $(t)$ provided $x_{\nu-k^{\prime}}$ $<t_{\nu}<x_{\nu}$, or

$$
t_{v}<x_{v}
$$$$
\nu=1, \cdots, n,
$$

since $k^{\prime}>n$ implies that $x_{\nu-k^{\prime}}=-\infty$. On the other hand we certainly have $D_{2}=\operatorname{det}\left\|\Lambda_{2}\left(t_{i}-y_{j}\right)\right\|>0$ if

$$
y_{1}<t_{1}<y_{2}<t_{2}<\cdots<y_{n}<t_{n} .
$$

Indeed $\Lambda_{2}(x)>0$ if $x>0$ and $\Lambda_{2}(x)=0$ if $x<0$, so that $D_{2}$ has positive elements on the main diagonal and only zero elements above it. Now if (1.23) hold, it is clear that increasing $t_{\nu}$ can be found satisfying (1.24) and (1.25), which completes the proof. The case when $k=0, h=\infty$, may be reduced to the previous one and leads to the reverse of (1.23), namely

$$
x_{\nu}<y_{\nu}, \quad \nu=1, \cdots, n .
$$

Proof of Case 1 for $k=h=\infty$. Let again $\Lambda=\Lambda_{1} * \Lambda_{2}$, where $\Lambda_{1}$ corresponds to the negative $\delta_{\nu}$ and $\Lambda_{2}$ to the positive ones. In (1.14), $D_{1}>0$ provided $x_{\nu}<t_{\nu}$, by (1.26), and $D_{2}>0$ provided $y_{v}<t_{\nu}$, by (1.23). Thus $D>0$ always, as was to be shown.

Proof of Case 1 for $h=\infty, k$ finite. With the same decomposition as before the positivity of (1.14) requires $x_{\nu}<t_{\nu}$, by (1.26), and $t_{\nu-k}<y_{\nu}<t_{\nu}$, by (1.13). Increasing $t_{\nu}$ will satisfy both conditions provided $x_{\nu}<y_{\nu+k}$, or

$$
x_{v-k}<y_{v}, \quad \nu=1, \cdots, n,
$$


which agree with (1.2). This completes a proof of Case 1 of our theorem.

\section{A proof of Theorem 1, Case 2}

5. This case requires some information on the behavior of Pólya frequency functions $\lambda(x)$ of small variance. We know that $\lambda(x)$ attains its maximal value at just one point $\left(^{(5)} x=\mu\right.$ and that $\lambda(x)$ is nonincreasing for $x \geqq \mu$ and nondecreasing for $x \leqq \mu$.

Lemma 2. Let $\left\{\lambda_{m}(x)\right\}$ be an infinite sequence of Pólya frequency functions such that

$$
\begin{array}{cl}
\int_{-\infty}^{\infty} \lambda_{m}(x) d x & =1, \quad \int_{-\infty}^{\infty} x \lambda_{m}(x) d x=0, \\
\sigma_{m}^{2}=\int_{-\infty}^{\infty} x^{2} \lambda_{m}(x) d x \rightarrow 0 & \text { as } m \rightarrow \infty .
\end{array}
$$

If $x=\mu_{m}$ denotes the point where $\lambda_{m}(x)$ attains its maximal value, then

$$
\begin{aligned}
\lim \mu_{m} & =0, \\
\lim \lambda_{m}\left(\mu_{m}\right) & =+\infty,
\end{aligned}
$$

Proof. Clearly, for a fixed $\eta>0$,

$$
\sigma_{m}{ }^{2}=\int x^{2} \lambda_{m} d x \geqq \int_{|x| \geqq \eta} x^{2} \lambda_{m} d x \geqq \eta^{2} \int_{|x| \geqq \eta} \lambda_{m} d x
$$

hence by the last relation (1.27):

$$
\int_{|x| \geqq \eta} \lambda_{m} d x \rightarrow 0 \text { as } m \rightarrow \infty, \quad \text { for every } \eta>0 .
$$

By the first relation (1.27) we conclude that

$$
\int_{-\eta}^{\eta} \lambda_{m} d x \rightarrow 1 \text { as } m \rightarrow \infty, \quad \text { for every } \eta>0 .
$$

But then clearly

$$
\max _{-\eta \geqq x \leqq \eta} \lambda_{m}(x) \rightarrow \infty \quad \text { as } \quad m \rightarrow \infty, \quad \text { for every } \eta>0 .
$$

We may now prove (1.28): If $x=\xi_{m}$ is a point where $\lambda_{m}(x)$ attains its maximum in $[-\eta, \eta]$, then by $(1.32)$

(5) This follows easily from Theorem 10, page 154, of Hirschman and Widder in [3]. Their $\lambda(x)=G_{m}(x)$ are not the most general Polya frequency functions, since $\gamma=0$ in their case. However, in Art. 6 below we are applying Lemma 2 to a case where $\gamma$ happens to vanish. 


$$
-\eta \leqq \xi_{m} \leqq \eta, \quad \lim _{m \rightarrow \infty} \lambda_{m}\left(\xi_{m}\right)=+\infty .
$$

If (1.28) does not hold, then we would have $\mu_{m}>2 \eta$, say, for arbitrarily large values of $m=m_{r}$. Since $\lambda_{m}(x)$ is nondecreasing in the range $\left[\xi_{m}, \mu_{m}\right]$ we conclude that

$$
\int_{\xi_{m}}^{2 \eta} \lambda_{m}(x) d x \geqq \int_{\xi_{m}}^{2 \eta} d x \cdot \lambda_{m}\left(\xi_{m}\right) \geqq \eta \lambda_{m}\left(\xi_{m}\right),
$$

hence, by (1.33), that

$$
\int_{\xi_{m}}^{2 \eta} \lambda_{m}(x) d x \rightarrow \infty \text { as } m=m_{\nu} \rightarrow \infty,
$$

which is absurd. This proves (1.28), and (1.29) follows from (1.32).

To prove (1.30), we use (1.28) and choose $M=M(\eta)$ such that $\left|\mu_{m}\right|<\eta / 2$ if $m>M$. But then, $\lambda_{m}(x)$ being nonincreasing in $\left[\mu_{m}, \eta\right]$, we have

$$
\frac{\eta}{2} \lambda_{m}(\eta) \leqq \int_{\eta / 2}^{\eta} \lambda_{m}(x) d x
$$

where the last integral converges to zero by (1.31). Thus $\lim \lambda_{m}(\eta)=0$ and (1.30) is established since $\lambda_{m}(x)$ is nonincreasing for $x \geqq \eta$.

6. Proof of Case 2 if $\gamma=0, \delta_{\nu}>0(\nu=1,2, \cdots)$. Let the transform be

$$
\int_{-\infty}^{\infty} e^{-x s} \Lambda(x) d x=\prod_{\nu=1}^{\infty} \frac{e^{\delta_{\nu s}}}{1+\delta_{\nu} s}, \quad \delta_{\nu}>0, \sum_{1}^{\infty} \delta_{\nu}=\infty
$$

We are to show that

$$
D=\operatorname{det}\left\|\Lambda\left(x_{i}-y_{j}\right)\right\|>0
$$

always holds. Let $\Lambda=\Lambda_{1} * \Lambda_{2}$ where $\Lambda_{1}$ corresponds to the product of the first $m$ factors of (1.34) and $\Lambda_{2}$ to the remainder. Setting

$$
\phi_{m}=\sum_{1}^{m} \delta_{\eta}
$$

we have that

$$
\prod_{v=1}^{m} \frac{1}{1+\delta_{y} s}=\int_{-\infty}^{\infty} e^{-\left(x+\phi_{m}\right) \cdot \Lambda_{1}}(x) d x=\int_{-\infty}^{\infty} e^{-\varepsilon x} \Lambda_{1}\left(x-\phi_{m}\right) d x .
$$

Now

$$
\operatorname{det}\left\|\Lambda_{1}\left(x_{i}-t_{j}\right)\right\|=\operatorname{det}\left\|\Lambda_{1}\left(x_{i}+\phi_{m}-t_{j}-\phi_{m}\right)\right\|
$$

and the transform (1.36) shows that $\Lambda_{1}\left(x-\phi_{m}\right)$ is a function to which Lemma 1 applies. Thus 


$$
D_{1}=\operatorname{det}\left\|\Lambda_{1}\left(x_{i}-t_{j}\right)\right\|>0
$$

provided $x_{\nu-m}+\phi_{m}<t_{\nu}<x_{p}+\phi_{m}, \nu=1, \cdots, n$. If $m \geqq n$, these conditions reduce to

$$
t_{\nu}<x_{\nu}+\phi_{m}, \quad \quad \nu=1, \cdots, n .
$$

Since $\phi_{m}=\sum_{1}^{m} \delta_{\nu} \rightarrow \infty$, we see that (1.38) are satisfied in an arbitrarily large cube of the $t$-space, if only $m$ is sufficiently large.

The transform of $\Lambda_{2}(x)$ is

$$
\int_{-\infty}^{\infty} e^{-x s} \Lambda_{2}(x) d x=\prod_{\nu=m+1}^{\infty} \frac{e^{\delta_{\nu} s}}{1+\delta_{\nu} s}=1+\frac{s^{2}}{2} \sum_{m+1}^{\infty} \delta_{\nu}^{2}+\cdots
$$

showing that $\lambda_{m}(x)=\Lambda_{2}(x)$ is a sequence of Pólya frequency functions satisfying the assumptions of Lemma 2, with

$$
\sigma_{m}^{2}=\sum_{\nu=m+1}^{\infty} \delta_{\nu}{ }^{2}
$$

In order to prove (1.35), we turn again to (1.14). Choose a fixed $\eta$ such that

$$
0<\eta<\frac{1}{2} \min \left(y_{\nu+1}-y_{\nu}\right)
$$

and let $t_{i}=y_{i}+\mu_{m}, i=1, \cdots, n$, where $\left\{\mu_{m}\right\}$ is the sequence of maximum points in Lemma 2 . We claim that for these values

$$
D_{2}=\operatorname{det}\left\|\Lambda_{2}\left(t_{i}-y_{j}\right)\right\|>0
$$

if $m>M$.

Indeed, if $i \neq j$ and $m$ is sufficiently large, we have

$$
\begin{aligned}
\left|t_{i}-y_{j}\right| & =\left|y_{i}-y_{j}+\mu_{m}\right| \\
& \geqq\left|y_{i}-y_{j}\right|-\left|\mu_{m}\right|>\left|y_{i}-y_{j}\right|-\eta>2 \eta-\eta=\eta,
\end{aligned}
$$

so that

$$
\lim _{m \rightarrow \infty} \Lambda_{2}\left(t_{i}-y_{j}\right)=0
$$

by $(1.30)$. On the other hand

$$
\Lambda_{2}\left(t_{i}-y_{i}\right)=\Lambda_{2}\left(\mu_{m}\right) \rightarrow \infty, \quad \text { as } m \rightarrow \infty,
$$

by (1.29). Thus $D_{2} \rightarrow \infty$, as $m \rightarrow \infty$, which amply proves (1.39). Now the $t_{v}$, as defined above, are bounded, in view of (1.28). Therefore the inequalities (1.38) are also satisfied by our $t_{i}$, if only $m$ is sufficiently large. Now (1.37), (1.39), and (1.14) imply (1.35), which completes the proof.

The remaining possibilities of Case 2 are now easily taken care of. Let us assume the transform (1.34), $\sum\left|\delta_{\nu}\right|=\infty$, but allow $\delta_{\nu}$ of either sign. To fix 
the ideas, let the negative $\delta_{v}$ have a divergent sum, and let again $\Lambda=\Lambda_{1} * \Lambda_{2}$, $\Lambda_{1}$ and $\Lambda_{2}$ corresponding to the negative and positive $\delta_{\nu}$ respectively. Then, in (1.14), we have $D_{1}>0$ always. Not so for $D_{2}$; however, the following is now clear: To whichever of the previously discussed cases the question of

$$
D_{2}=\left\|\Lambda_{2}\left(t_{i}-y_{j}\right)\right\|>0
$$

may belong, given the $y_{\nu}$, we can always find such increasing $t_{\nu}$ as to make $D_{2}>0$. As $D_{1}>0,(1.14)$ shows that $D>0$.

Finally, to treat the last remaining case, let

$$
\int_{-\infty}^{\infty} e^{-x s} \Lambda(x) d x=e^{\gamma^{2}} \cdot F(s), \quad \gamma>0,
$$

where $F(s)$ is of either of the forms (8) or (1.34). Let for the last time $\Lambda$ $=\Lambda_{1} * \Lambda_{2}$, the transforms of these factors being the two factors of (1.40), respectively. The reasoning of the last paragraph again applies. In (1.14) we have $D_{1}>0$ for any $t_{\nu}\left({ }^{6}\right)$, while by all previous cases, increasing $t_{i}$ may be found as to produce $D_{2}>0$. Thus $D>0$ and a proof of Theorem 1 is completed.

\section{INTERPOLATION BY SPLINE CURVES}

7. The problem. Let

$$
\xi_{1}<\xi_{2}<\cdots<\xi_{n}
$$

be $n$ given points and $k$ a natural integer. Let $F(x)$ be defined in each of the intervals $\left(-\infty, \xi_{1}\right),\left(\xi_{1}, \xi_{2}\right), \cdots,\left(\xi_{n},+\infty\right)$ by a separate polynomial, of degree not exceeding $k$, in such a way that the composite function $F(x)$ be continuous, for all real $x$, together with its first $k-1$ derivatives. $F^{(k)}(x)$ is a step-function with possible discontinuities at the points (2.1). A function $F(x)$ of this kind is called a spline function $\left({ }^{7}\right)$ (or curve) of degree $k$, while the points (2.1) are referred to as its knots.

The truncated function (1.4) and its powers are well suited for the representation of spline functions. Indeed, it is easily seen that

$$
F(x)=P_{k}(x)+\sum_{\nu=1}^{n} A_{\nu}\left(x-\xi_{\nu}\right)_{+}^{k},
$$

where $P_{k}(x)$ is an arbitrary polynomial of degree at most $k$ and $A_{v}$ are arbitrary parameters, represents uniquely the most general spline curve of degree $k$ having the knots (2.1). Thus $F(x)$ depends on $n+k+1$ arbitrary parameters.

(6) See [6, Art. 3, Example 2].

(7) See $[5$, p. 67], for the connection with the flexible rod, called a spline, used for fitting smooth curves to experimental data. 
We may now formulate our problem as follows: Let

$$
x_{1}<x_{2}<\cdots<x_{n+k+1}
$$

be a given set of $n+k+1$ abscissae; when can we interpolate in the points (2.3) an arbitrary given set of ordinates $Y_{1}, Y_{2}, \cdots, Y_{n+k+1}$, by means of our spline curve (2.2)?

8. Its solution. As a first step we observe the following: If we choose in any way $k+1$ fixed abscissae $y_{1}, \cdots, y_{k+1}$ such that

$$
y_{1}<y_{2}<\cdots<y_{k+1}<x_{1}, \quad y_{k+1}<\xi_{1},
$$

then the polynomial $P_{k}(x)$, of $(2.2)$, may be uniquely written as

$$
P_{k}(x)=\sum_{\nu=1}^{k+1} c_{\nu}\left(x-y_{\nu}\right)^{k}
$$

for appropriate values of the $c_{v}$. But then we also have

$$
P_{k}(x)=\sum_{\nu=1}^{k+1} c_{\nu}\left(x-y_{\nu}\right)_{+}^{k}, \quad \text { if } x \geqq y_{k+1} .
$$

If we now write

$$
y_{k+2}=\xi_{1}, y_{k+3}=\xi_{2}, \cdots, y_{n+k+1}=\xi_{n},
$$

and correspondingly define $c_{\nu+k+1}=A_{\nu}(\nu=1, \cdots, n)$, we see that the arbitrary spline curve (2.2) of knots (2.1) may also be written in the form

$$
F(x)=\sum_{\nu=1}^{n+k+1} c_{\nu}\left(x-y_{\nu}\right)_{+}^{k}, \quad \text { in the range } x \geqq y_{k+1} .
$$

We conclude that the interpolation problem of the previous article has a solution if and only if the determinant of order $n+k+1$

$$
\operatorname{det}\left\|\left(x_{i}-y_{j}\right)_{+}^{k}\right\| \neq 0 .
$$

By Corollary $1, \S 1$, this is the case provided

$$
x_{\nu-k-1}<y_{\nu}<x_{\nu} \quad(\nu=1,2, \cdots, n+k+1) .
$$

In view of (2.4) and (2.3) we see that the first $k+1$ inequalities (2.8) are automatically satisfied. Returning by (2.6) to our old notation we find the remaining $n$ inequalities $(2.8)$ to be

$$
\begin{aligned}
& x_{1}<\xi_{1}<x_{k+2}, \\
& x_{2}<\xi_{2}<x_{k+3}, \\
& . . . . . \\
& x_{n}<\xi_{n}<x_{n+k+1} .
\end{aligned}
$$


We state the result as

ThEOREM 2. We can interpolate in $n+k+1$ given abscissae (2.3) arbitrarily given ordinates, by a spline curve of degree $k$ and knots (2.1), if and only if the inequalities (2.9) are satisfied.

REMARK. The inequalities (2.9) may be described in words: The first $n$ interpolation points $x_{1}, \cdots, x_{n}$ precede the knots $\xi_{1}, \cdots, \xi_{n}$, respectively, which in turn precede the last $n$ interpolation points $x_{k+2}, \cdots, x_{n+k+1}$, respectively. Notice that all these conditions are satisfied automatically in case the knots $\xi_{1}, \cdots, \xi_{n}$ are chosen among the $n+k-1$ interpolation points $x_{2}, x_{3}, \cdots, x_{n+k}$.

We rephrase this last result as follows:

THEOREM 3. Let there be given a set of $N+1$ abscissae

$$
x_{0}<x_{1}<\cdots<x_{N}
$$

and $N+1$ arbitrary corresponding ordinates $y_{\nu}(\nu=0, \cdots, N) . A s$ is well known, we may interpolate them, uniquely, by a polynomial of degree at most $N$. This corresponds to the classical case $n=0$ of no knots whatever.

Also we may choose any combination of $n(1 \leqq n \leqq N-1)$ among the interior abscissae

$$
x_{1}, x_{2}, \cdots, x_{N-1} \text {, }
$$

to serve as the knots $\xi_{1}, \cdots, \xi_{n}$ of an interpolating spline curve $y=F(x)$, in which case again we may interpolate uniquely by a spline curve of degree

$$
k=N-n \text {. }
$$

REMARKs. 1. In the extreme case of $n=N-1$, all points (2.11) are knots of $F(x)$, which, by (2.12), is a spline curve of degree $k=N-(N-1)=1$. Therefore $y=F(x)$ is the ordinary polygonal line obtained by linear interpolation between each pair of consecutive points. Theorem 3 furnishes a sequence of interpolation procedures bridging the gap between the ordinary polynomial interpolation and the linear polygonal interpolation, each additional knot lowering the degree of the spline curve by one unit.

2. To each of these procedures there corresponds an interpolation formula expressing the interpolating spline curve $F(x)$ in terms of the ordinates $y_{\nu}$, with coefficients which are the "fundamental functions" of the procedure. In the case of $N=4, n=1$ and therefore $k=3$, with

$$
x_{0}=-2, x_{1}=-1, x_{2}=0, x_{3}=1, x_{4}=2 \text { and } \xi_{1}=0 \text {, }
$$

we obtain the formula

$$
F(x)=y_{0} l_{0}(x)+y_{1} l_{1}(x)+y_{2} l_{2}(x)+y_{-1} l_{1}(-x)+y_{-2} l_{2}(-x),
$$

with the following expressions for the fundamental functions: 


$$
\begin{aligned}
& l_{0}(x)=(1+x)\left(1+\frac{x}{2}\right)\left(1-\frac{3}{2} x\right)+\frac{3}{2} x_{+}^{3} \\
& l_{1}(x)=\frac{1}{3} x(x+1)(x+2)-x_{+}^{3} \\
& l_{2}(x)=-\frac{1}{24} x(x+1)(x+2)+\frac{1}{4} x_{+}^{3}
\end{aligned}
$$

In (2.13) we have changed our notation for $y_{v}$ in order to exhibit the symmetry of this formula.

3. We conclude by raising the following question. Let $f(x)$ be given as continuous in the range $[a, b]$ which we divide into $N$ equal parts by the points (2.10). It is well known that the interpolating polynomial of degree $N$ does not always converge to $F(x)$, as $N \rightarrow \infty\left(^{8}\right)$. However, the polygonal interpolating spline curve $(k=1)$ always does. Is there a sequence of our new procedures, with $k=N-n \rightarrow \infty$ as $N \rightarrow \infty$, producing a spline curve $F(x)$ of degree $k$, which will always converge to $f(x)$ in $[a, b]$ ? And how strongly may $k \rightarrow \infty$ with $N$, subject to this condition? Similar questions can be raised concerning the quadrature formulae obtained by integrating our interpolation formulae. Further problems will undoubtedly occur to the reader.

\section{REFERENCES}

1. F. Gantmakher and M. Krein, Oscillatory matrices and kernels and small vibrations of mechanical systems (in Russian), 2d ed., Moscow, 1950.

2. H. Hahn, Über das Interpolationsproblem, Math. Zeit. vol. 1 (1918) pp. 115-142.

3. I. I. Hirschman and D. V. Widder, The inversion of a general class of convolution transforms, Trans. Amer. Math. Soc. vol. 66 (1949) pp. 135-201.

4. M. Krein and G. Finkelstein, Sur les fonctions de Green complètement non-négatives des operateurs differentiels ordinaires, C. R. (Doklady) Acad. Sci. URSS. vol. 24 (1939) pp. 220-223.

5. I. J. Schoenberg, Contributions to the problem of approximation of equidistant data by analytic functions, Parts A and B, Quarterly of Applied Mathematics vol. 4 (1946) pp. 45-99, 112-141.

6. - On Polya frequency functions I. The totally positive functions and their Laplace transforms, Journal d'Analyse Mathématique vol. 1 (1951) pp. 331-374.

7. I. J. Schoenberg and Anne Whitney, Sur la positivite des determinants de translations des fonctions de frequence de Polya avec une application au probleme d'interpolation par les fonctions "spline," C. R. Acad. Sci. Paris vol. 228 (1949) pp. 1996-1998.

University of Pennsylvania, Philadelphia, Pa.

(8) See [2] concerning the general problem mentioned and also for further references. 